DOI: 10.12731/2227-930X-2020-2-48-57

УДК 004.94

\title{
ПРОБЛЕМЫ ПОСТАВОК ЗАПАСНЫХ ЧАСТЕЙ ОТОПИТЕЛЬНОГО ОБОРУДОВАНИЯ
}

Преображенский Ю.П., Питолин М.В.

В статье рассматривается задача, связанная с поставками запасных частей. Решение ее базируется на статистическом подходе. Приведень результаты проведенного моделирования.

Ключевые слова: система перевозок; система поставок; управление; моделирование.

\section{PROBLEMS OF DELIVERY OF SPARE PARTS OF HEATING EQUIPMENT}

\section{Preobrazhenskiy Yu.P., Pitolin M.V.}

The paper discusses the problem associated with the supply of spare parts. Its decision is based on a statistical approach. The results of the simulation are presented.

Ключевые слова: transportation system; supply system; management; modeling.

\section{Введение}

В настоящее время идет развитие организаций, связанных с производством и распространением отопительного оборудования. При этом для решения задач на практике могут быть использованы разные методы, в том числе - статистические. В данной работе рассматриваются возможности построения соответствующих моделей и получения на их основе результатов.

Основные методы статистического анализа потоков заказов на запасные детали отопительного оборудования. Корреляционный 
анализ, в основном, применяется для исследования взаимосвязи отдельных показателей и характеристик процессов управления ремонтом и техническим обслуживанием с учетом решений по планированию запасами запасных деталей, узлов и агрегатов.

При исследовании совокупности показателей объемов заказов, корреляционный анализ определяет структуру попарных зависимостей. Например, благодаря ему, при ремонте или обслуживании автомобиля, может быть проверена гипотеза о связанности запасных деталей.

Оценкой ковариации случайных величин является выборочная ковариация, представленная смещенной, которая рассчитывается как $\tilde{k}_{x y}=\frac{1}{n} \sum_{i=1}^{n}\left(x_{i}-\bar{x}\right)\left(y_{i}-\bar{y}\right)$. Получить несмещенную оценку возможно с помощью математического ожидания равного $M \tilde{k}=\frac{n-1}{n} k_{x y}$. На основании соотношения вычисляется дисперсия оценки ковариации:

$$
D \tilde{k}=\frac{n\left(\mu_{22}-\mu_{11}^{2}\right)}{(n-1)^{2}}+\frac{2 \mu_{22}-\mu_{20} \mu_{02}-3 \mu_{11}^{2}}{(n-1)^{2}}+\frac{\mu_{22}-\mu_{20} \mu_{02}-2 \mu_{11}^{2}}{n(n-1)^{2}}
$$

Величина $\hat{k}_{x y}=\frac{1}{n-1} \sum_{i=1}^{n}\left(x_{i}-\bar{x}\right)\left(y_{i}-\bar{y}\right)$ применяется в качестве несмещенной и состоятельной оценки. Корреляционный анализ дает возможности для того, чтобы определять некоторые взаимозависимости. При этом на его базе не всегда есть возможности для того, чтобы была показана структура взаимозависимостей по каждому из показателей. При таких условиях может быть полезным применение факторного анализа. При его использовании возникают возможности для того, чтобы была определена структура взаимозависимостей среди общей совокупности показателей. Какие будут основные шаги в алгоритме? Прежде всего, происходит определение главных компонент. На их основе происходит определение того, как первоначальная система показателей будет прямым образом преобразована к абстрактной, распределенной относительно информативности. Затем приступают к обратному преобразованию. После этого строят аналитическую 
зависимость между основными компонентами и группой общих факторов. Тогда можно говорить об особенностях взаимосвязей и характеристиках соответствующих показателей. Факторный анализ требуется для того, чтобы определять зависимость временных рядов, связанных с потоками, и требований по запасным деталям, отделенных от действующих факторов в результате корреляционного анализа. Главные задачи: с целью последующей классификации поиск структурной зависимости между отдельными показателями и снижение размерности пространства исследуемых факторов.

Реализуемый анализ зависимости переменных, с привлечением статистического пакета, возникает на основе следующей модели:

$$
\xi_{1}=\sum_{j=1}^{m} \lambda_{1 j} F_{j}+\varepsilon_{1}, \ldots, \xi_{p}=\sum_{j=1}^{m} \lambda_{1 p j} F_{j}+\varepsilon_{p},
$$

$F_{1}, F_{2}, \ldots, F_{p}$ - общие факторы, причем $D F_{i}=1 \operatorname{cov}\left(F_{1}, F_{J}\right)=0 \quad i \neq j$;

$\lambda_{1 p j}-$ факторные нагрузки $i, j=1 . . m, m<p$;

$D \varepsilon_{i}=\tau_{i}, i=1 . . p, \tau_{i}$ специфическая дисперсия;

$\varepsilon_{1}, \ldots, \varepsilon_{p}-$ специфические факторы, причем $\operatorname{cov}\left(\varepsilon_{i}, \varepsilon_{j}\right)=0 \quad i \neq j$.

Учет общих и специфических факторов осуществляется в качестве независимых. Однако при этом необходимо соблюдать выполнение условия $\operatorname{cov}\left(F_{i}, \varepsilon_{j}\right)=0 \quad i=1 . . m ; \quad j=1 . . p$.

Реализация ковариаций исходных переменных и факторизации дисперсий представлена в модели следующим образом:

$$
\begin{gathered}
\sigma_{i j}-\lambda_{i 1}+\ldots+\lambda_{i m} \lambda_{j m} . i \neq j, \\
\sigma_{i j}^{2}-\lambda_{i 1}^{2}+\ldots+\lambda^{2}{ }_{i m}+\tau_{i}, i=1 . . p .
\end{gathered}
$$

Из следующих задач состоит методика осуществления факторного анализа: 1. В один набор данных объединяются все переменные; 2. Выполняется нахождение линии, на которой дисперсия набора будет максимальной (определение первого фактора); 3. Производится поиск второй линии, на которой остающийся разброс выступает максимальным, и так далее.

Конечным результатом является последовательная система не коррелированных абстрактных факторов. 


\section{Результаты проведенного моделирования}

Проведем анализ, интенсивности влияния в отношении критериальной функции параметров, находящихся под контролем. Принято решение использовать в качестве целевой функции, функцию издержек (4). Рассмотрим таблицу 1 , на которой приведены ценовые показатели на определенные виды деталей для отопительных котлов модели Вахі. Плата за хранение деталей $\mathrm{s}$ составляет 1-1,5\%, если ориентироваться на стоимость деталей. Каким образом назначать штраф за дефицит? Его величина $h$ рассматривается в виде срочная доставки. Она будет находиться в границах 300-800 рублей. Параметры, связанные с геометрическим распределением по деталям «термоизолирующая панель» и «вентилятор» были получены на первом шаге. В качестве единственного параметра геометрического распределения можно считать значение р. Относительно него и были осуществлены расчеты (рис. 1).

Таблицุа 1.

Стоимость запасных частей Вахі

\begin{tabular}{|c|l|l|l|c|}
\hline № & \multicolumn{1}{|c|}{ Деталь } & Цена & Группа & Код \\
\hline 1 & Термоизолирующая панель & 722,04 & 1 & 005213380 \\
\hline 2 & Вентилятор & 6123,3 & 1 & 005682150 \\
\hline 3 & Проводка электрическая 230 B & 1496,3 & 1 & 008611810 \\
\hline 4 & Пневмореле Есо Classic 24F & 629,68 & 1 & $57008821 P$ \\
\hline 5 & Основной теплообменник LUNA-3 COMFORT & 9170,8 & 2 & 000620870 \\
\hline 6 & Электронная плата (Ноnеywell) & 8107,42 & 2 & 005669670 \\
\hline 7 & Гидрогруппа с краном подпитки & 735,47 & 3 & 711666100 \\
\hline 8 & Насос циркуляционный 1 SPEED 6МТ & 8505 & 2 & 721957300 \\
\hline 9 & Основной теплообменник 24 кВт с клипсами & 9674 & 3 & 005632470 \\
\hline 10 & Трубка теплообменник/трехходовой клапан & 738,32 & 3 & 005676870 \\
\hline 11 & Вторичный теплообменник на 10 пластин & 4249,0 & 3 & 005686660 \\
\hline 12 & Крепёжные клипсы теплообменника & 83,00 & 3 & 005113650 \\
\hline
\end{tabular}




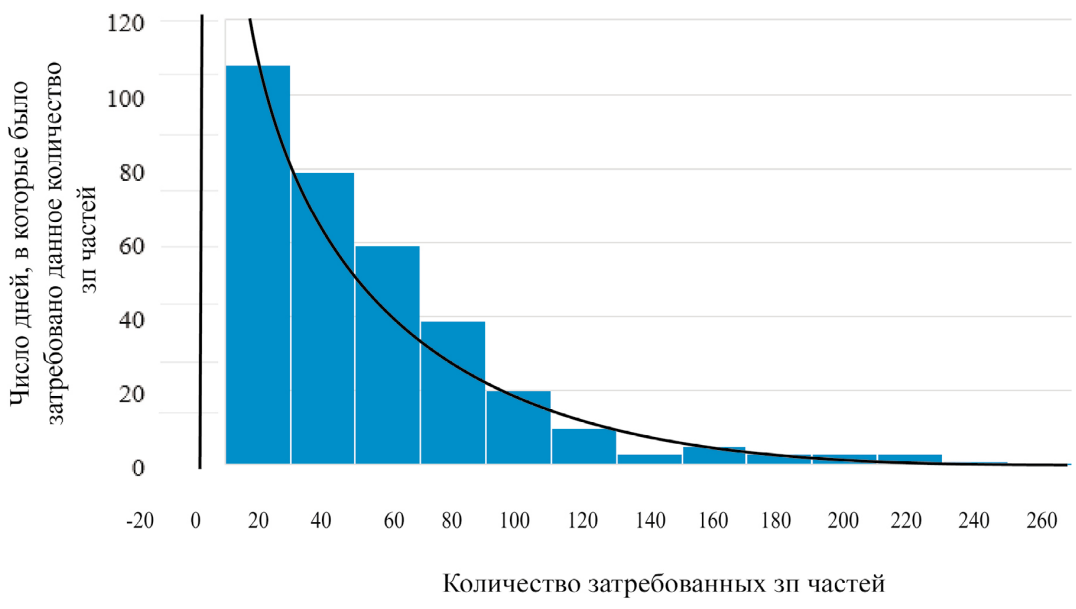

а) Термоизолирующая панель

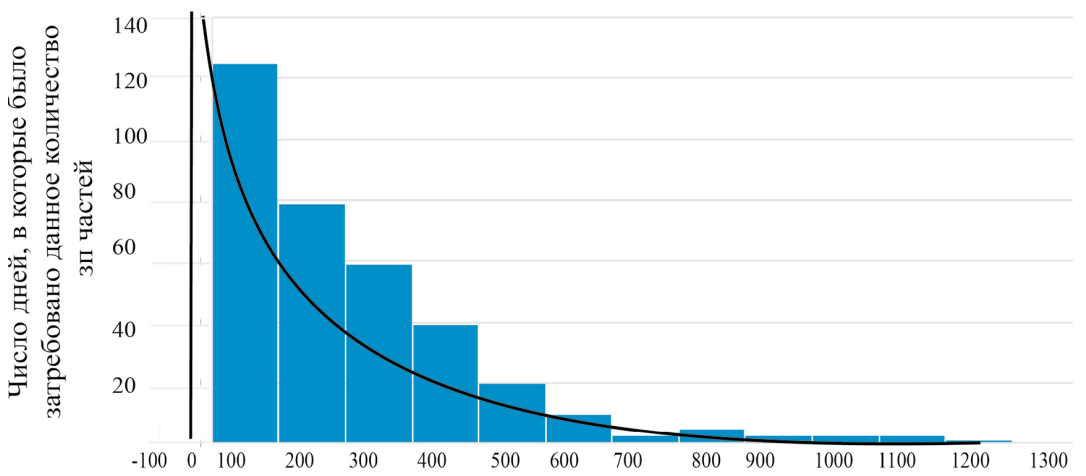

Количество затребованных зп частей

б) Вентилятор

Рис. 1. Геометрическое распределение. Оценка потока заявок на темоизолирующую панель и вентилятор

В качестве базовых значений можно считать значения $\mathrm{p}$, и еще плату, связанную с хранением s, а также дефицит $\mathrm{h}$. При этом анализируются однопродуктовые схемы управления запасами в номенклатурах деталей. 
Расшифровка обозначений

Таблицча 2.

\begin{tabular}{|c|l|}
\hline Tmod & \multicolumn{1}{|c|}{ Значение горизонта планирования, дни } \\
\hline Zmin & Точка заказа, ед \\
\hline Zmax & Значение максимального объема заказа, ед \\
\hline Tzak & Значение времени выполнения заказа, дни \\
\hline s & Значение платы, связанной с хранением, руб/месс \\
\hline g & Значение платы, связанной с доставкой, руб \\
\hline h & Значение штрафа, связанного с отсутствием, руб/ед \\
\hline p & $\begin{array}{l}\text { Значение параметра в ряде расходов, рассматривается геометрическое } \\
\text { распределение }\end{array}$ \\
\hline
\end{tabular}

Далее рассмотрим полученные результаты, где в таблицах 3-9 приведены параметры моделирования. Рассмотрим влияние параметров на целевую функцию издержек запасной части со средней интенсивностью расхода, основного теплообменника.

Таблицุа 3.

Таблица, иллюстрирующая параметры моделирования в основном теплообменнике

\begin{tabular}{|c|c|c|c|c|c|c|c|}
\hline $\begin{array}{c}\text { Tmod, } \\
\text { Дни }\end{array}$ & $\begin{array}{c}\text { Zmin, } \\
\text { Ед }\end{array}$ & $\begin{array}{c}\text { Zmax, } \\
\text { ед }\end{array}$ & $\begin{array}{c}\text { Tzak, } \\
\text { дни }\end{array}$ & $\begin{array}{c}\text { s, } \\
\text { руб./мес. }\end{array}$ & g, руб. & $\begin{array}{c}\text { h, } \\
\text { руб./ед. }\end{array}$ & p \\
\hline 365 & - & 20 & 2 & 20 & 2 & 300 & 0,216 \\
\hline
\end{tabular}

Таблицุа 4.

Таблица, иллюстрирующая параметры моделирования по основному теплообменнику, когда варьируется Zmax

\begin{tabular}{|c|c|c|c|c|c|c|c|}
\hline $\begin{array}{c}\text { Tmod, } \\
\text { Дни }\end{array}$ & $\begin{array}{c}\text { Zmin, } \\
\text { Ед }\end{array}$ & $\begin{array}{c}\text { Zmax, } \\
\text { ед }\end{array}$ & $\begin{array}{c}\text { Tzak, } \\
\text { дни }\end{array}$ & $\begin{array}{c}\text { s, } \\
\text { руб./мес. }\end{array}$ & g, руб. & $\begin{array}{c}\text { h, } \\
\text { руб./ед. }\end{array}$ & p \\
\hline 365 & 18 & - & 2 & 20 & 2 & 300 & 0,216 \\
\hline
\end{tabular}

Таблицуа 5.

Таблица, иллюстрирующая параметры моделирования по основному теплообменнику, когда варьируется $h$

\begin{tabular}{|c|c|c|c|c|c|c|c|}
\hline $\begin{array}{c}\text { Tmod, } \\
\text { Дни }\end{array}$ & $\begin{array}{c}\text { Zmin, } \\
\text { Ед }\end{array}$ & $\begin{array}{c}\text { Zmax, } \\
\text { ед }\end{array}$ & $\begin{array}{c}\text { Tzak, } \\
\text { дни }\end{array}$ & $\begin{array}{c}\text { s, } \\
\text { руб./мес. }\end{array}$ & g, руб. & $\begin{array}{c}\text { h, } \\
\text { руб./ед. }\end{array}$ & p \\
\hline 365 & 18 & 75 & 2 & 20 & 2 & - & 0,216 \\
\hline
\end{tabular}

Таблицча 6.

Таблица, иллюстрирующая параметры моделирования по основному теплообменнику для Z $\mathrm{min}$, когда увеличивается h с 300 до 700 руб.

\begin{tabular}{|c|c|c|c|c|c|c|c|}
\hline $\begin{array}{c}\text { Tmod, } \\
\text { Дни }\end{array}$ & $\begin{array}{c}\text { Zmin, } \\
\text { Ед }\end{array}$ & $\begin{array}{c}\text { Zmax, } \\
\text { ед }\end{array}$ & $\begin{array}{c}\text { Tzak, } \\
\text { дни }\end{array}$ & $\begin{array}{c}\text { s, } \\
\text { руб./мес. }\end{array}$ & g, руб. & $\begin{array}{c}\text { h, } \\
\text { руб./ед. }\end{array}$ & p \\
\hline 365 & 18 & 75 & 2 & 20 & 2 & - & 0,216 \\
\hline
\end{tabular}


Таблица, иллюстрирующая параметры моделирования по основному теплообменнику, когда варьируется Z $\max$ с условием увеличения штрафа h c 300 до 700 руб.

\begin{tabular}{|c|c|c|c|c|c|c|c|}
\hline $\begin{array}{c}\text { Tmod, } \\
\text { Дни }\end{array}$ & $\begin{array}{c}\text { Zmin, } \\
\text { Ед }\end{array}$ & $\begin{array}{c}\text { Zmax, } \\
\text { ед }\end{array}$ & $\begin{array}{c}\text { Tzak, } \\
\text { дни }\end{array}$ & $\begin{array}{c}\text { s, } \\
\text { руб./мес. }\end{array}$ & g, руб. & $\begin{array}{c}\mathrm{h}, \\
\text { руб./ед. }\end{array}$ & p \\
\hline 365 & 30 & - & 2 & 20 & 2 & 700 & 0,215 \\
\hline
\end{tabular}

Как видно, увеличение h обуславливает рост параметров Zmin с 20 до 30, a Zmax с 80 до 90 единиц. Затем проведено рассмотрение моделирования издержек деталей при том, что есть высокая интенсивность расхода - электронной платы.

Таблица 8.

Таблица, иллюстрирующая параметры моделирования по электронной плате, когда варьируется Zmin

\begin{tabular}{|c|c|c|c|c|c|c|c|}
\hline $\begin{array}{c}\text { Tmod, } \\
\text { Дни }\end{array}$ & $\begin{array}{c}\text { Zmin, } \\
\text { Ед }\end{array}$ & $\begin{array}{c}\text { Zmax, } \\
\text { ед }\end{array}$ & $\begin{array}{c}\text { Tzak, } \\
\text { дни }\end{array}$ & $\begin{array}{c}\text { s, } \\
\text { руб./мес. }\end{array}$ & g, руб. & $\begin{array}{c}\mathrm{h}, \\
\text { руб./ед. }\end{array}$ & p \\
\hline 365 & - & 2000 & 2 & 7 & 1 & 300 & 0,006 \\
\hline
\end{tabular}

Таблица 9.

Таблица, иллюстрирующая параметры моделирования по электронной плате, когда варьируется Zmax

\begin{tabular}{|c|c|c|c|c|c|c|c|}
\hline $\begin{array}{c}\text { Tmod, } \\
\text { Дни }\end{array}$ & $\begin{array}{c}\text { Zmin, } \\
\text { Ед }\end{array}$ & $\begin{array}{c}\text { Zmax, } \\
\text { ед }\end{array}$ & $\begin{array}{c}\text { Tzak, } \\
\text { дни }\end{array}$ & $\begin{array}{c}\text { s, } \\
\text { руб./мес. }\end{array}$ & g, руб. & $\begin{array}{c}\mathrm{h}, \\
\text { руб./ед. }\end{array}$ & p \\
\hline 365 & 400 & 2 & 7 & 7 & 1 & 300 & 0,006 \\
\hline
\end{tabular}

Видно, что существует различие по оптимальным параметрам точки заказа и максимального заказа. Это определяется тем, какая номенклатура деталей, это вытекает из характеристик спроса на них, а также соответствующих цен.

\section{Вывод}

Таким образом, в результате проведенного анализа показана необходимость в том, чтобы были разработаны формальные модели и соответствующие алгоритмов оптимизации управляемых параметров. Кроме того, продемонстрировано, что для описания задачи следует опираться на применение моделей планирования эксперимента для оценки влияния вариации сразу всеми факторами, а также перехода на много продуктовую модель, исходя из того, что была показана взаимосвязь по поставкам среди всех деталей. 
Информация о конфликте интересов. Авторы заявляют об отсутствии конфликта интересов.

Информация о спонсорстве. Исследование не имело спонсорской поддержки.

\section{Сиисок литературь}

1. Тухватуллин Б.Т., Левченко Д.В., Стебайлов М.Ю. Формирование структурно-энергетических параметров рабочих поверхностей высокоточных деталей при эксплуатации автомобильного транспорта // International Journal of Advanced Studies. 2019. T. 9. № 3. С. 64-70.

2. Лысанов Д.М., Пономаренко Н.А. Количественная оценка уровня качества оборудования // International Journal of Advanced Studies. 2018. Т. 8. № 4-2. С. 56-61.

3. Лысанов Д.М., Бикмухаметова Л.Т. Анализ показателей качества и конкурентоспособности оборудования // International Journal of Advanced Studies. 2018. T. 8. № 4-2. С. 50-55.

4. Гуськова Л.Б. О построении автоматизированного рабочего места менеджера // Успехи современного естествознания. 2012. № 6. С. 106.

5. Самойлова У.А. О некоторых характеристиках управления предприятием // Вестник Воронежского института высоких технологий. 2014. № 12. С. 176-179.

6. Пеньков П.В. Экспертные методы улучшения систем управления // Вестник Воронежского института высоких технологий. 2012. № 9. С. $108-110$.

7. Максимов И.Б. Принципы формирования автоматизированных рабочих мест // Вестник Воронежского института высоких технологий. 2014. № 12. С. 130-135.

8. Максимов И.Б. Классификация автоматизированных рабочих мест // Вестник Воронежского института высоких технологий. 2014. № 12. C. 127-129.

\section{References}

1. Tuhvatullin B.T., Levchenko D.V., Stebajlov M.Yu. International Journal of Advanced Studies. 2019. V. 9. № 3, pp. 64-70. 
2. Lysanov D.M., Ponomarenko N.A. International Journal of Advanced Studies. 2018. V. 8. № 4-2, pp. 56-61.

3. Lysanov D.M., Bikmuhametova L.T. International Journal of Advanced Studies. 2018. V. 8. № 4-2, pp. 50-55.

4. Gus'kova L.B. Uspekhi sovremennogo estestvoznaniya. 2012. № 6. P. 106.

5. Samojlova U.A. Vestnik Voronezhskogo instituta vysokih tekhnologij. 2014. № 12, pp. 176-179.

6. Pen'kov P.V. Vestnik Voronezhskogo instituta vysokih tekhnologij. 2012. № 9, pp. 108-110.

7. Maksimov I.B. Vestnik Voronezhskogo instituta vysokih tekhnologij. 2014. № 12, pp. 130-135.

8. Maksimov I.B. Vestnik Voronezhskogo instituta vysokih tekhnologij. 2014. № 12, pp. 127-129.

\section{ДАННЫЕ ОБ АВТОРАХ}

Преображенский Андрей Петрович, профессор, доктор технических наук, доцент

Автономная некоммерческая образовательная организация высшего образования Воронежский институт высоких технологий

ул. Ленина, 73a, г. Воронеж, 394043, Российская Федераиุия

gorbenkoon@yandex.ru

Питолин Михаил Владимирович, доцент, кандидат технических наук, доцент

Федеральное государственное казенное образовательное учреждение выстего образования «Воронежский институт Министерства внутренних дел Российской Федераuุuu»»

nр-m Патриотов, 53, г. Воронеж, 394065, Российская Федерачия gorbenkoon@yandex.ru 


\section{DATA ABOUT THE AUTHORS}

Preobrazhenskiy Andrey Petrovich, Professor, Doctor of Technical Sciences, Associate Professor Voronezh Institute of High Technologies

73a, Lenin Str., Voronezh, 394043, Russian Federation gorbenkoon@yandex.ru

ORCID: 0000-0002-6911-8053

Pitolin Mikhail Vladimirovich, associate Professor, Candidate of Technical Sciences, Associate Professor Voronezh Institute of the Ministry of Internal Affairs of the Russian Federation

53, Patriotov Ave., Voronezh, 394065, Russian Federation gorbenkoon@yandex.ru 